\title{
Prediction of effective moduli of carbon nanotube-reinforced composites with waviness and debonding
}

\author{
L.H. Shao ${ }^{\text {a,b }}$, R.Y. Luo ${ }^{\text {c }}$, S.L. Bai ${ }^{\text {b,d }}$, J. Wang ${ }^{\text {b,* }}$ \\ ${ }^{a}$ Institute of Mechanics, Chinese Academy of Sciences, Beijing 100080, PR China \\ ${ }^{\mathrm{b}}$ LTCS and Department of Mechanics and Aerospace Engineering, College of Engineering, Peking University, Beijing 100871, PR China \\ ${ }^{\mathrm{c}}$ School of Science, Beihang University, Beijing 100083, PR China \\ ${ }^{\mathrm{d}}$ Department of Advanced Materials and Nanotechnology, College of Engineering, Peking University, Beijing 100871, PR China
}

Available online 19 February 2008

\begin{abstract}
Carbon nanotubes (CNTs) have been regarded as ideal reinforcements of high-performance composites with enormous applications. However, the waviness of the CNTs and the interfacial bonding condition between them and the matrix are two key factors that influence the reinforcing efficiency. In this paper, the effects of the waviness of the CNTs and the interfacial debonding between them and the matrix on the effective moduli of CNT-reinforced composites are studied. A simple analytical model is presented to investigate the influence of the waviness on the effective moduli. Then, two methods are proposed to examine the influence of the debonding. It is shown that both the waviness and debonding can significantly reduce the stiffening effect of the CNTs. The effective moduli are very sensitive to the waviness when the latter is small, and this sensitivity decreases with the increase of the waviness.
\end{abstract}

(c) 2008 Elsevier Ltd. All rights reserved.

Keywords: Carbon nanotube; Composite; Waviness; Debonding; Effective modulus

\section{Introduction}

Carbon nanotubes (CNTs) with exceptional stiffness and strength have been regarded as ideal reinforcements of composites. Enormous efforts and studies have been devoted to the synthesis, characterization, modeling and simulation of CNT-reinforced nanocomposites [1,2]. Many encouraging experimental results have been reported. For example, the CNT/alumina nanocomposites show an enhanced hardness and toughness compared to monolithic materials [3]. Qian et al. [4] reported CNT-reinforced polystyrene with good dispersion and CNT-matrix adhesion. The elastic modulus and the strength are improved significantly over that of the matrix with only a very small fraction of the CNTs.

On the other hand, it is also widely recognized that the experimental mechanical properties of CNT-reinforced

\footnotetext{
${ }^{*}$ Corresponding author. Tel.: +86106275 7948; fax: +86 1062751812 . E-mail address: jxwang@pku.edu.cn (J. Wang).
}

nanocomposites are quite different from the theoretical expectation $[5,6]$. There are also many analyses about the causes for the difference, including waviness [4-10], agglomeration $[3,10]$, weak bonding between the matrix and the CNTs $[6,8,10]$, etc. However, the effects of these defects on the effective mechanical properties of CNT-reinforced nanocomposites have not been well understood and modeled. It is noted that Fisher et al. [9] investigated the effect of waviness on the effective stiffness, by assuming a sinusoidal waviness and using an approach combining numerical (finite element) computation and the MoriTanaka scheme. Shi et al. [10] studied the effects of agglomeration and waviness on the effective moduli of CNT-reinforced composites, while assuming that the CNTs are in a helical shape. But according to experimental observations $[4,7,8]$, the waviness of the CNTs may not be as severe as helical. Anumandla and Gibson [11] developed a micromechanics model for estimating the effective elastic moduli of carbon nanotube-reinforced composites incorporating the waviness and random orientation of the nano- 
tubes. Their model is based on combining the predictions of the effective elastic constants of composites containing continuous wavy fibres by Chan and Wang [12], and Hsiao and Daniel [13], and the model of Christensen and Waals [14] that relates the elastic constants of a unidirectional fiberous composite and those of a composite with randomly orientated fibres. Considering the huge difference between the stiffnesses and strengths of the CNTs and most of the matrix materials, the load transfer at the interface is a critical factor that decides the reinforcing efficiency of the CNTs. There have been many studies on the interface loadtransferring mechanisms [15-17]. Also due to the huge difference of the stiffness and the complicated mechanisms at the interface, weak bonding areas are quite likely to exist, and debonding may occur at very low level of loading or even during manufacturing. While the effect of partial debonding in traditional particle-reinforced composites on their effective properties has been well revealed $[18,19]$, the effect of partial debonding between the CTNs and matrices on the properties of the composites has not been well studied.

In this paper, first, we propose a simple model to predict the effect of the waviness of CNTs on the effective elastic constants of the nanocomposites. Then, we present two simple methods to examine the effect of partial debonding on the effective moduli. In the analyses, as in other theoretical studies in the literature [20-22], the CNTs are treated as solid short fibres. The curved CNTs and those with partial debonding are replaced by equivalent straight short fibres, and then the effective moduli are calculated using the Mori-Tanaka scheme. Therefore, the models and the derived formulas are simple and easy to use.

\section{Micromechanics model for curved CNTs}

Many experimental observations have shown that CNTs generally exist in a curved shape in the nanocomposites $[4,7,8]$. To simplify the analysis we will treat the nanotubes as solid short fibres with a circular cross-section and exhibiting a bow-like waviness, as shown in Fig. 1. Here $a$ and $\lambda$ are the amplitude (vertical projection) and half-wavelength (horizontal projection) of a curved CNT, respectively. The diameter and the volume fraction of the CNTs are denoted by $d$ and $f_{r}$. It is obvious that a curved CNT will have reinforcing effects both in the direction of the chord and in the perpendicular. Therefore, in order to simulate the reinforcing effect of a curved CNT in a composite, it is projected onto the vertical and horizontal directions, respectively, as shown in Fig. 2. Then we replace the curved fibre by one straight fibre along the chord, and two another straight fibres in the perpendicular direction. The length and the volume fraction of the chord fibre are denoted by $\lambda$ and $f_{2}$, respectively. The length and the volume fraction of the fibres in the perpendicular direction are denoted by $a$ and $f_{3}$, respectively. The diameters of all the projected fibres are assumed to be the same as that of the original curved fibre, denoted by $d$.

Considering a representative volume $V$ of a CNT-reinforced composite, then we have the relation

$f_{2}=N \frac{\pi d^{2} \lambda}{4 V}, \quad f_{3}=2 \cdot N \frac{\pi d^{2} a}{4 V}$

where $N$ is the number of the CNTs. Denoting the waviness index by $\frac{a}{\lambda}=\delta$, from Eq. (1), we get

$\frac{f_{2}}{f_{3}}=\frac{1}{2 \delta}$

$f_{2}+f_{3}=f_{r}$

From Eqs. (2) and (3), we get the volume fractions as follows:

$f_{2}=\frac{f_{r}}{1+2 \delta}, \quad f_{3}=\frac{f_{r}}{1 / 2 \delta+1}$

Next, we need to calculate the effective elastic constants of the projected equivalent fibres, namely, we shall replace the curved fibre by a straight equivalent fibre in the chord direction under the condition that the curved fibre and the straight one have the same reinforcing effect in the chord direction. For this, we assume that the effective modulus of the horizontally projected fibre in Fig. 2 is $E_{f 2}$, and the effective modulus of a composite which contains these uniaxial fibres is $E_{x}$. From the rules-of-mixtures we get $E_{f 2} f_{2}+E_{\mathrm{m}} f_{\mathrm{m}}=E_{x}$, then

$E_{f 2}=\frac{E_{x}-E_{\mathrm{m}} f_{\mathrm{m}}}{f_{2}}$

where $E_{\mathrm{m}}$ is Young's modulus of the matrix, $f_{\mathrm{m}}$ and $f_{2}$ are the volume fractions of the matrix and the fibres with the length $\lambda$, respectively. It is noted that there have been some studies predicting the effective moduli of composites reinforced by continuous curved fibres [12-14,23,24]. We adopt the result of the effective longitudinal modulus in Ref. [23]

$$
E_{x}=\frac{(1+c)^{3 / 2}}{\left(1+\frac{c}{2}\right) S_{11}-\left[1+\frac{3 c}{2}-(1+c)^{3 / 2}\right] S_{22}+\frac{c}{2}\left(2 S_{12}+S_{66}\right)}
$$

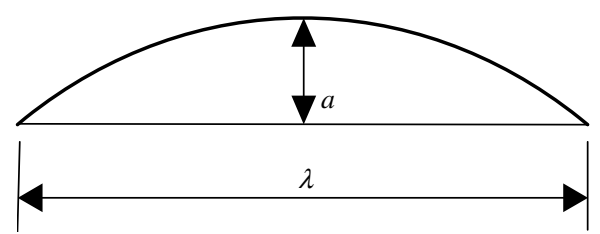

Fig. 1. The model of a curved CNT.

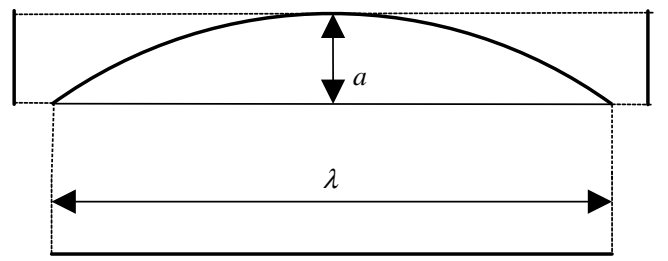

Fig. 2. The projection of a curved CNT. 
where

$c=\left(\frac{\pi a}{\lambda}\right)^{2}=\pi^{2} \delta^{2}$

$\left.\begin{array}{l}S_{11}=\frac{1}{E_{L}} ; S_{22}=\frac{1}{E_{T}} \\ S_{12}=-\frac{v_{L T}}{E_{L}} ; S_{66}=\frac{1}{G_{L T}}\end{array}\right\}$

In the above formulas, $E_{L}, E_{T}$ and $G_{L T}$ are the longitudinal, transverse and shear moduli of the composite with the uniaxial straight fibres. $v_{L T}$ is the longitudinal-transverse Poisson's ratio. To simplify the analysis we will calculate these parameters using the rules-of-mixtures

$$
\left.\begin{array}{l}
E_{L}=f_{f} E_{f}+f_{\mathrm{m}} E_{\mathrm{m}} \\
E_{T}=\left(\frac{f_{f}}{E_{f}}+\frac{f_{\mathrm{m}}}{E_{\mathrm{m}}}\right)^{-1} \\
v_{L T}=f_{f} v_{f}+f_{\mathrm{m}} v_{\mathrm{m}} \\
G_{L T}=f_{f} G_{f}+f_{\mathrm{m}} G_{\mathrm{m}}
\end{array}\right\}
$$

where $E_{\mathrm{m}}, E_{f}, G_{\mathrm{m}}, G_{f}$ and $v_{\mathrm{m}}, v_{f}$ are Young's moduli and Poisson's ratios of the matrix and the fibres. $f_{\mathrm{m}}$ and $f_{f}$ are the volume fractions of the matrix and the effective fibres, respectively. Substituting Eqs. (6)-(10) into Eq. (5) we obtain the effective modulus of the horizontally projected equivalent fibre.

Similarly, assuming that the effective modulus of the vertically projected fibre in Fig. 2 is $E_{f 3}$, and the effective modulus of a composite containing such straight uniaxial fibres is $E_{y}$. From the rules-of-mixtures we get $2 E_{f 3} f_{3}+$ $E_{\mathrm{m}} f_{\mathrm{m}}=E_{y}$, and

$E_{f 3}=\frac{E_{y}-E_{\mathrm{m}} f_{\mathrm{m}}}{2 f_{3}}$

where $E_{\mathrm{m}}$ is Young's modulus of the matrix, $f_{\mathrm{m}}$ and $f_{3}$ are the volume fractions of the matrix and the fibres with the length $a$, respectively. Kuo et al. [24] previously predicted the the effective transverse modulus of a composite containing curved fibres having the sinusoidal waviness

$E_{y}=\frac{(1+c)^{3 / 2}}{\left[(1+c)^{3 / 2}-1-\frac{3 c}{2}\right] S_{11}+\left(1+\frac{c}{2}\right) S_{22}+\frac{c}{2}\left(2 S_{12}+S_{66}\right)}$

Substituting Eqs. (7)-(10) and (12) into Eq. (11) we can obtain the effective modulus of the vertically projected equivalent fibre.

Now, the curved fibre has been replaced by two kinds of equivalent straight fibre whose volume fractions and effective elastic constants have been known. In this paper, we consider the nanocomposites in which the CNTs are randomly distributed and orientated. Then we calculate the effective moduli of the curved CNT-reinforced nanocomposite using the Mori-Tanaka method, with the properties of the above-obtained equivalent straight fibres and taking into account the average over the orientations of the fibres [25-27],
$\mathbf{C}=\mathbf{C}_{1}+\sum_{r=2}^{M} v_{r}\left\{\left(\mathbf{C}_{r}-\mathbf{C}_{1}\right): \mathbf{H}_{r}\right\}:\left[\sum_{r=1}^{M} v_{r}\left\{\mathbf{H}_{r}\right\}\right]^{-1}$

where $\mathbf{C}$ denotes the effective modulus tensor of the composite, and $M$ denotes the number of the types of the reinforcements. The volume fraction of the $r$ th phase is denoted by $v_{r}$. Curly brackets $\{\bullet\}$ represent an average over all possible orientations. The tensor $\mathbf{H}_{r}$ is

$\mathbf{H}_{r}=\left[\mathbf{I}+\mathbf{S}_{r}: \mathbf{C}_{1}^{-1}:\left(\mathbf{C}_{r}-\mathbf{C}_{1}\right)\right]^{-1}$

where $\mathbf{I}$ denotes the fourth-order symmetric unit tensor, $\mathbf{C}_{1}$ and $\mathbf{C}_{r}$ denote the tensors of elastic moduli of the matrix and the $r$ th phase, respectively, and $\mathbf{S}_{r}$ is the Eshelby tensor corresponding to the $r$ th phase which is simulated by a prolate spheroid. When the CNTs are randomly orientated, the composite exhibits isotropy. In this case, the stiffness tensor $\mathbf{C}$ in Eq. (14) degenerates into a fourth-order isotropic tensor which can be written as

$\mathbf{C}=3 K \mathbf{J}+2 \mu \mathbf{K} \equiv(3 K, 2 \mu)$

where $K$ and $\mu$ are the effective bulk and shear moduli of the composite, and $\mathbf{J}$ and $\mathbf{K}$ are two fourth-order projection tensors. The details of the Mori-Tanaka scheme can be found in many references, e.g. [26,27], and thus they are not reproduced here. In particular, it has been widely recognized that for stiff inclusions, the Mori-Tanaka method can give fairly accurate prediction for low volume fractions of the inclusions [27].

Fisher et al. [9] have modeled a wavy CNT by a sinusoidal curved fibre. In order to analyze the effect of the curved shape, we consider three cases: arc of a circle, sinusoid and parabola, respectively. For these wavinesses, we calculate the respective effective moduli of the composites containing curved fibres and the corresponding straight fibres which have the same lengths as the curved ones. In order to compare the present predictions with those of Fisher et al. [9], all the calculations are based on the parameters in Table 1, where $E_{\mathrm{ERM}}$ is the so-called effective reinforced modulus of a straight fibre that replaces a curved CNT given by Fisher et al. [9] using the finite element computation for a sinusoidal wavy CNT. Thus, $E_{\text {ERM }}$ can also be used in the MoriTanaka micromechanical scheme to calculate the effective elastic moduli of the composite.

Fig. 3 shows the variation of the non-dimensionalized effective Young's modulus $E / E_{\mathrm{m}}$ with the volume fraction $f_{r}$ of the CNTs. For the considered small waviness, the values for the three different wavy shapes mentioned above are almost identical, which means that in this case the shape has little effect on the effective elastic moduli. It is seen that the effective Young's modulus predicted by the present model is very close to the result predicted by using $E_{\mathrm{ERM}}$ in

Table 1

Elastic constants and geometry parameters [9]

\begin{tabular}{llllll}
\hline$E_{\mathrm{m}}(\mathrm{GPa})$ & $E_{\mathrm{NT}}(\mathrm{GPa})$ & $E_{\mathrm{ERM}}(\mathrm{GPa})$ & $a / \lambda$ & $\lambda / d$ & $v_{\mathrm{m}}=v_{\mathrm{NT}}$ \\
\hline 2.25 & 450 & 383 & 0.05 & 60 & 0.30 \\
\hline
\end{tabular}




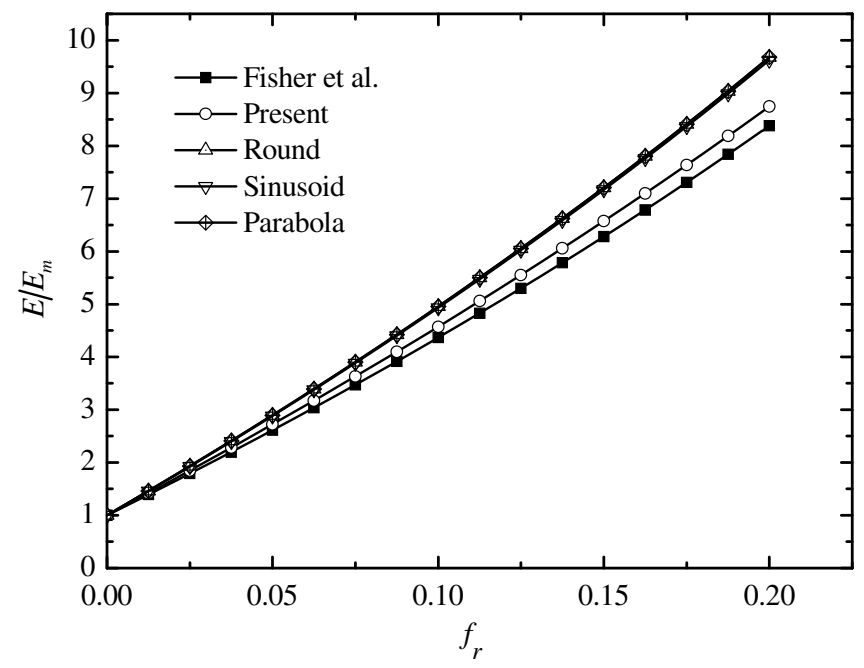

Fig. 3. Variation of effective Young's modulus with volume fraction.

the Mori-Tanaka scheme. However, the present prediction is slightly higher than that of the latter. The reason for this difference may be due to that in the model of Fisher et al. [9] a wavy CNT is replaced by only one straight fibre in the chord direction, whereas in the present model, the wavy CNT is replaced by three fibres, namely, one in the chord direction and two in the perpendicular direction (Fig. 2).

Figs. 4 and 5 show the variations of the non-dimensionalized effective bulk modulus $K / K_{1}$ and shear modulus $\mu / \mu_{1}$ with the waviness $\delta\left(\delta=\frac{a}{\lambda}\right)$ when the volume fraction $f_{r}=0.1$. It is seen that when $\delta<0.4$, the effective moduli of the composite decrease drastically with the increase of $\delta$. When $\delta>0.4$, the variation is not significant. Therefore, for the considered material, the reinforcing efficiency of the CNTs is very sensitive to the waviness when it is less than about 0.4 .

\section{Micromechanics model for debonding of CNTs}

Properties of the fibre-matrix interface in composites have been of scientific and technological interest for a long

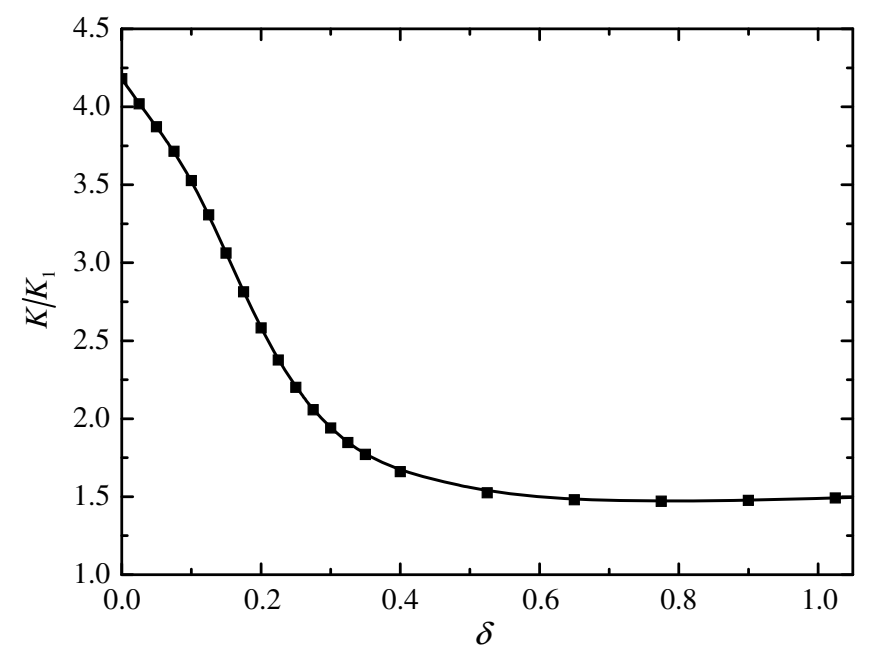

Fig. 4. Variation of effective bulk modulus $K / K_{1}$ with waviness $\delta$.

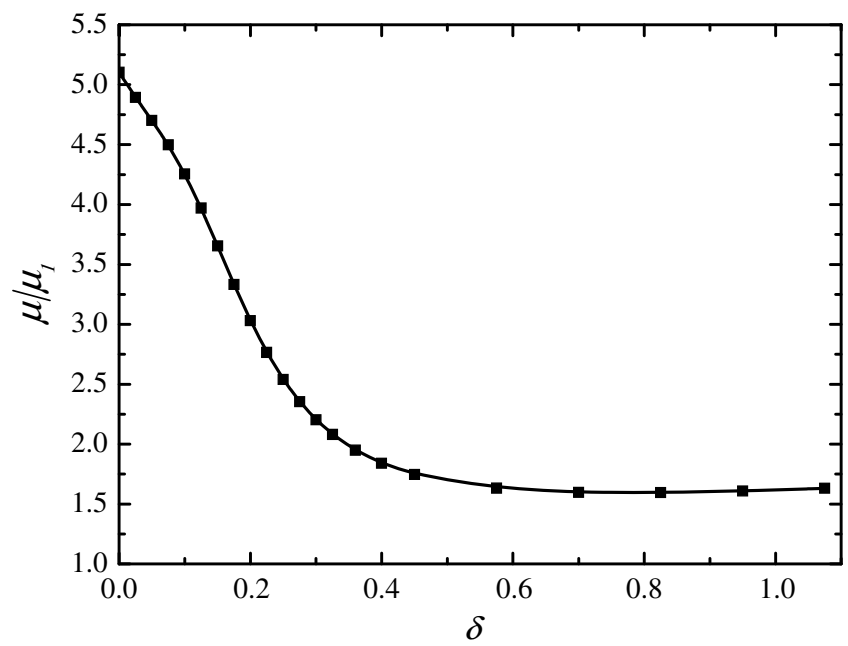

Fig. 5. Variation of effective shear modulus $\mu / \mu_{1}$ with waviness $\delta$.

time, because interfacial debonding intensely affects the fracture toughness [28,29], yield strength [30], and other properties of composites. Debonding can result in early void formation, and also ductile matrix failure can be promoted by the stress and strain concentrations occurring around sharp fibre edges [31]. When the interfacial strength is relatively weak and a two-phase system is under a triaxial tension, interfacial debonding is likely to be the principal mode of damage process. Upon continuous loading such a process is a progressive one, so that as more and more interfacial debonding takes place, the composite will lose its load-carrying capacity and the overall stiffness $[18,19]$. Weak interfacial bonding and debonding will greatly affect the overall properties of CNT-reinforced nanocomposites. In this section, we will present a model to forecast the effective moduli of composites reinforced by randomly orientated short fibres, which have debonded along the axial direction. Then we will analyze the effect of the debonding extent and other factors on the effective moduli.

\subsection{Methods and theory}

We consider a representative volume $V$ of a composite which contains $N$ short fibres, among which there are $N_{p}$ perfectly-bonded fibres and $N_{d}$ partially debonded and fully debonded fibres so that $N=N_{p}+N_{d}$. Here the subscript $p$ denotes perfectly bonded and $d$ denotes partially debonded and fully debonded fibres. For a single fibre with partial interfacial debodning, as shown in Fig. 6, the length and radius of the fibre are denoted by $L$ and $r_{f}$, respectively. Assuming that the debonding length, and the area of the fibre cross-section are $l_{d}$, and $A$, respectively. We denote $x=\frac{l_{d}}{L}, y=\frac{N_{p}}{N}$.

We propose two methods to analyze the effect of debonding. In the first method, we divide a debonded fibre into two parts: the debonded part and the bonded part, and then treat the two parts separately. Thus, there are 4 phases in the composite, namely, the matrix (the 1st phase), the 


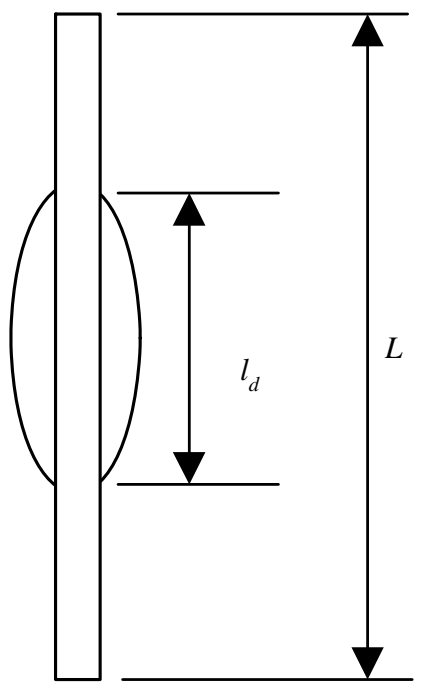

Fig. 6. Debonding of interface between a fibre and matrix.

perfectly bonded fibres (the 2nd phase), the perfectly bonded part of the debonded fibres (the 3rd phase) and the fully debonded part of the debonded fibres (the 4th phase). The volume fractions and the aspect ratios of the three reinforcing phases are as follows:

(1) The 2nd phase: the volume fraction is $f_{2}=N_{p} \cdot \frac{A L}{V}=f_{r} \cdot y$, and the aspect ratio is $\zeta_{2}=L /$ $2 r_{f} \equiv \zeta . f_{r}=\frac{N A L}{V}$ is the volume fraction of all fibres in the composite.

(2) The 3rd phase: the volume fraction is $f_{3}=N_{d} \cdot \frac{A\left(L-l_{d}\right)}{V}=f_{r} \cdot(1-x)(1-y)$, and the aspect ratio is $\zeta_{3}=\frac{L-l_{d}}{2 r_{f}}=\zeta \cdot(1-x)$.

(3) The 4th phase: the volume fraction is $f_{4}=N_{d} \cdot \frac{A l_{d}}{V}=f_{r} \cdot x(1-y)$, and the aspect ratio is $\zeta_{4}=\frac{l_{d}}{2 r_{f}}=\zeta \cdot x$.

A fibre itself is treated as an isotropic medium. Then, from the analysis, we know that the 2 nd and the 3 rd phases are isotropic, having the same property as the original intact fibre. However, because of the debonding, the in situ properties of the 4th phase must be reduced. Using the method in Ref. [19], the isotropic debonded inclusion is replaced by a fictitious transversely isotropic one, whose elastic properties are calculated in the following [19].

In order to deal with a transversely isotropic material, it is convenient to use Hill's stress-strain relations [32]

$$
\begin{aligned}
& \frac{1}{2}\left(\sigma_{22}+\sigma_{33}\right)=k\left(\varepsilon_{22}+\varepsilon_{33}\right)+l^{\prime} \varepsilon_{11} \\
& \sigma_{11}=l\left(\varepsilon_{22}+\varepsilon_{33}\right)+n \varepsilon_{11} \\
& \sigma_{22}-\sigma_{33}=2 m\left(\varepsilon_{22}-\varepsilon_{33}\right) \\
& \sigma_{23}=2 m \varepsilon_{23}, \sigma_{12}=2 p \varepsilon_{12}, \sigma_{13}=2 p \varepsilon_{13}
\end{aligned}
$$

where direction 1 is along the axis of symmetry, and plane $2-3$ is isotropic. Then, in Walpole's short-hand notations, the transversely isotropic elastic modulus tensor can be written as [33]
$\mathbf{C}=\left(2 k, n, 2 m, 2 p, l, l^{\prime}\right)$

Because tensor $\mathbf{C}$ is diagonally symmetric $\left(l=l^{\prime}\right)$, it can be further expressed as

$\mathbf{C}=(2 k, n, 2 m, 2 p, l, l)$

When the material is isotropic, tensor $\mathbf{C}$ reduces to

$\mathbf{C}=(2 k, k+\mu, 2 \mu, 2 \mu, k-\mu)$

The elastic constants $k, n, m, p, l$ are in Hill's notation. For isotropic case, they are related to the bulk and shear constants $K$ and $\mu$ through

$$
\begin{aligned}
& k=K+\frac{1}{3} \mu, \quad n=K+\frac{4}{3} \mu, \quad m=p=\mu, \\
& l=K-\frac{2}{3} \mu
\end{aligned}
$$

As shown in Fig. 6, the debonding takes place on the lateral 2-3 surface so that $\sigma_{22}=\sigma_{33}=0$. It follows from Eq. (16)

$\left(\varepsilon_{22}+\varepsilon_{33}\right)=-\frac{l_{0}}{k_{0}} \varepsilon_{11}, \quad \sigma_{11}=\left(n_{0}-\frac{l_{0}^{2}}{k_{0}}\right) \varepsilon_{11}$

The debonded part carries no shear stress, $\sigma_{12}=\sigma_{23}=0$. Thus, the elastic modulus of the fictitious transversely isotropic inclusion is

$\mathbf{C}_{4}^{*}=\left(0, n_{0}-\frac{l_{0}^{2}}{k_{0}}, 0,0,0,0\right)$

The modulus of an original intact fibre is denoted by the subscript " 0 ".

The analysis above fits the partially debonded case $(x<1)$. If a fibre is not fully debonded, the bonded part can still carry some stress $\sigma_{11}$, and thus Eq. (21) is reasonable. However, when a fibre is fully debonded $(x=1)$, it is difficult for the ends of the fibre to carry the axial load because of its small sectional area. In this case, the assumption $\sigma_{11}=0$ should be a better approximation. Considering that the load is transferred from the interface of the bonded part to the debonded part, and this transfer is related to the area of the bonded part, through a simple analysis [34], we multiply the modulus in Eq. (22) by a factor $\left(1-\frac{l_{d}}{L}\right)$ and get a modified modulus $(1-x)\left(n_{0}-\frac{l_{0}^{2}}{k_{0}}\right)$. Then Eq. (22) is changed to

$\mathbf{C}_{4}^{*}=\left(0,(1-x)\left(n_{0}-\frac{l_{0}^{2}}{k_{0}}\right), 0,0,0,0\right)$

Therefore, with the elastic constants and geometric parameters of the constituents known, we can predict the effective moduli of the composite using the Mori-Tanaka scheme for multi-phase composites.

In the above, we presented the first method to deal with the debonding, where a debonded fibre is divided into two phases. Here, we present the second method to model the effect of debonding. In this method, a single debonded fibre is simply replaced by one fictitious transversely isotropic fibre, so there are 3 phases in the composite, namely, the 
matrix (1st phase), the perfectly bonded fibres (2nd phase) whose properties are the same as the 2nd phase in the above first method, and the fictitious transversely isotropic fibres (3rd phase) which replaces the partially debonded fibres. The volume fraction and the aspect ratio of the 3rd phase are $v_{3}=N_{d} \cdot \frac{A L}{V}=f_{r}(1-y)$ and $\zeta_{2}=L / 2 r_{f} \equiv \zeta$, respectively. The elastic constants of the fictitious transversely isotropic fibres are calculated below.

Consider a fibre as shown in Fig. 6. We assume that the matrix carries the stress $\sigma_{11}$ remotely and the modulus of the fibre is much larger than that of the matrix. The load is transferred from the interface of the bonded part to the debonded part. Through a simple analysis [34], the effective longitudinal elastic modulus of the fictitious transversely isotropic fibre can be calculated by

$E_{1}^{f}=\left(1-\frac{l_{d}}{L}\right) E_{0}=(1-x) E_{0}$

The effective transverse elastic modulus is

$E_{2}^{f}=(1-x) E_{0}$

The effective longitudinal and transverse shear moduli are

$G_{12}^{f}=G_{23}^{f}=(1-x) G_{0}$

The plane-strain bulk modulus in the transversely isotropic plane is

$k_{23}^{f}=(1-x) k_{0}$

In the above formulas, the parameters with the subscript " 0 " denote those of the intact fibre. Eqs. (24)-(27) give the five elastic constants of the fictitious transversely isotropic fibre. Thus, we are ready to predict the effective elastic moduli of the composite that contains perfectly bonded and debonded short fibres using the Mori-Tanaka scheme.

\subsection{Numerical analysis}

For the case of debonding, we first calculate the effective elastic constants using the first method. The elastic modulus tensor of the intact fibre is $\mathbf{C}_{0}=\left(2 k_{0}, k_{0}+\mu_{0}, 2 \mu_{0}\right.$, $\left.2 \mu_{0}, k_{0}-\mu_{0}\right)$. We have $n_{4}=(1-x) \frac{\mu_{0}\left(3 k_{0}-\mu_{0}\right)}{k_{0}}, k_{4}=l_{4}=0$, $m_{4}=p_{4}=0$. The aspect ratio is assumed to be $\zeta=250$. The Young's modulus of the isotropic matrix and the carbon nanotubes are $E_{1}=20 \mathrm{GPa}[35]$ and $E_{0}=910 \mathrm{GPa}$ [36], respectively. The Poisson's ratio is $v_{1}=v_{0}=0.3$.

Fig. 7 shows the variation of $K / K_{1}$ with the volume fraction $f_{r}$, where $K$ and $K_{1}$ are the bulk modulus of the composite and the matrix, respectively. In the figure, curve $B$ shows the case when all fibres are perfectly bonded with the matrix; curve $C$ is for the case when fifty percent of the fibres are debonded by $20 \%$; curve $D$ is for the case when all fibres have debonded by $20 \%$; and curve $E$ is for the case when all fibres are fully debonded. The effective shear modulus $\mu / \mu_{1}$ exhibits the same variation, as shown in Fig. 8. In curve $E$, the effective moduli of the composite decrease with the increase of the volume fraction when all the fibres are fully debonded. This is because the fully

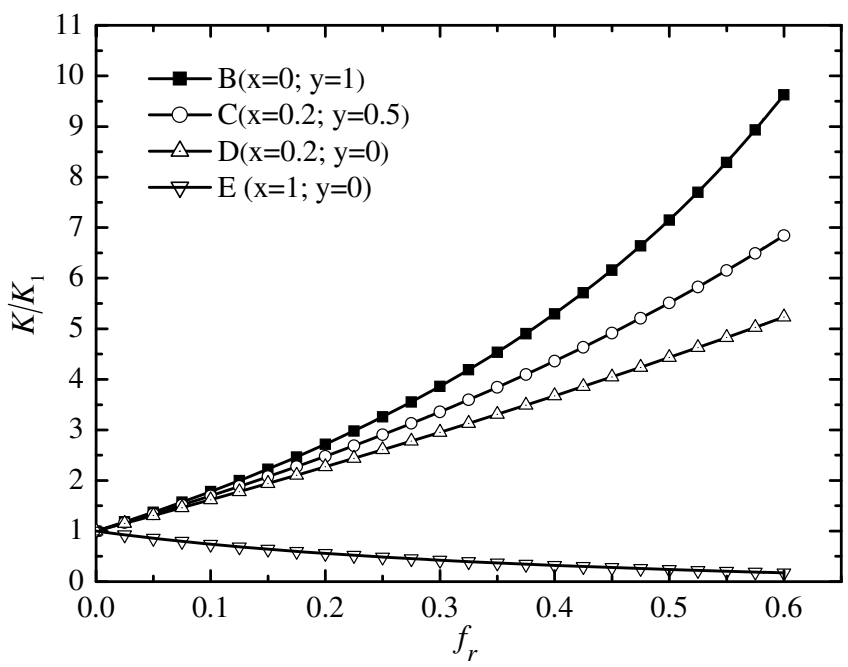

Fig. 7. Variation of effective bulk modulus with volume fraction.

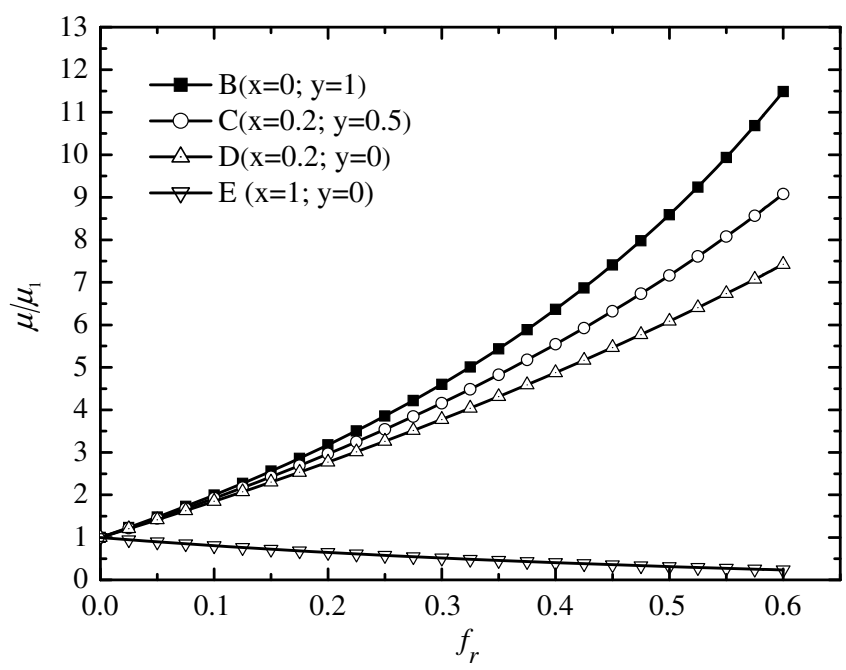

Fig. 8. Variation of effective shear modulus with volume fraction.

debonded fibres do not carry any load; they simply play the role of voids.

Fig. 9 depicts the variation of $K / K_{1}$ with the debonding extent $x$ for volume fraction $f_{r}=0.2$. All the fibres have the same debonding extent for curve $B$. There are fifty percent of fibres having the same debonding extent for curve $C$. All the fibres are perfectly bonded for curve $D$, and in this case the modulus does not depend on $x$. The effective shear modulus $\mu / \mu_{1}$ exhibits the same variation, as shown in Fig. 10.

Figs. 11 and 12 show the effective bulk modulus $K / K_{1}$ varying with the volume fraction $f_{r}$ when $x=0.2, y=0.5$ and $x=0.2, y=0$, respectively. The results that are obtained using the two methods dealing with the debonding are almost identical when the volume fraction of the fibre is below $40 \%$. It is also the case for the effective shear modulus $\mu / \mu_{1}$. Generally, the content of carbon nanotubes in current nanocomposites is much less than $40 \%$, so either 


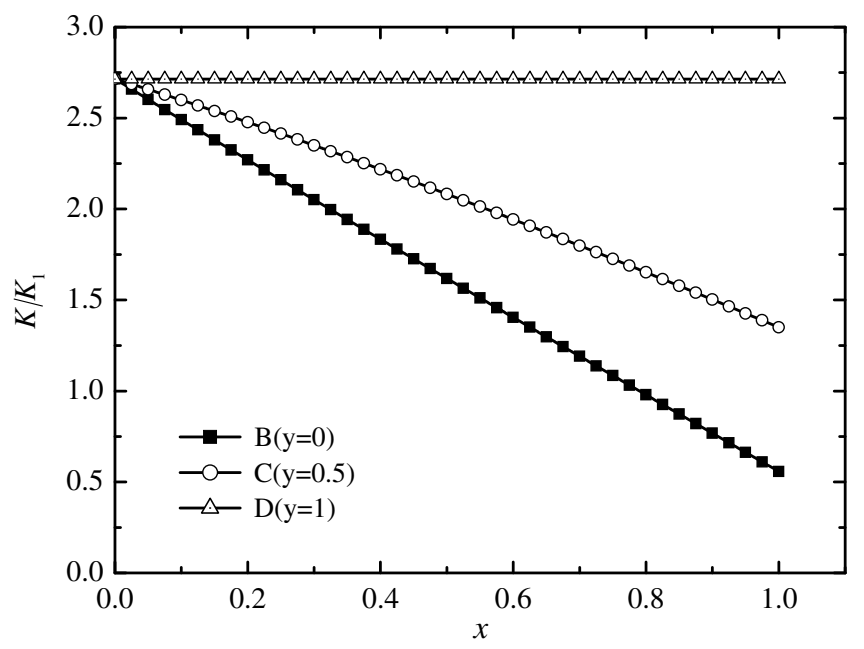

Fig. 9. Variation of effective bulk modulus with debonding extent $\left(f_{r}=0.2\right)$.

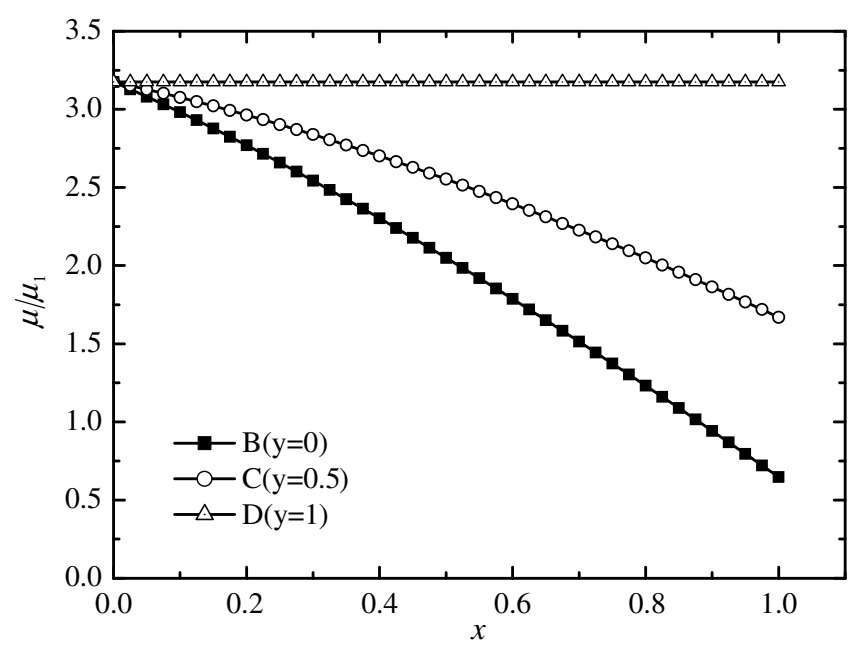

Fig. 10. Variation of effective shear modulus with debonding extent $\left(f_{r}=0.2\right)$.

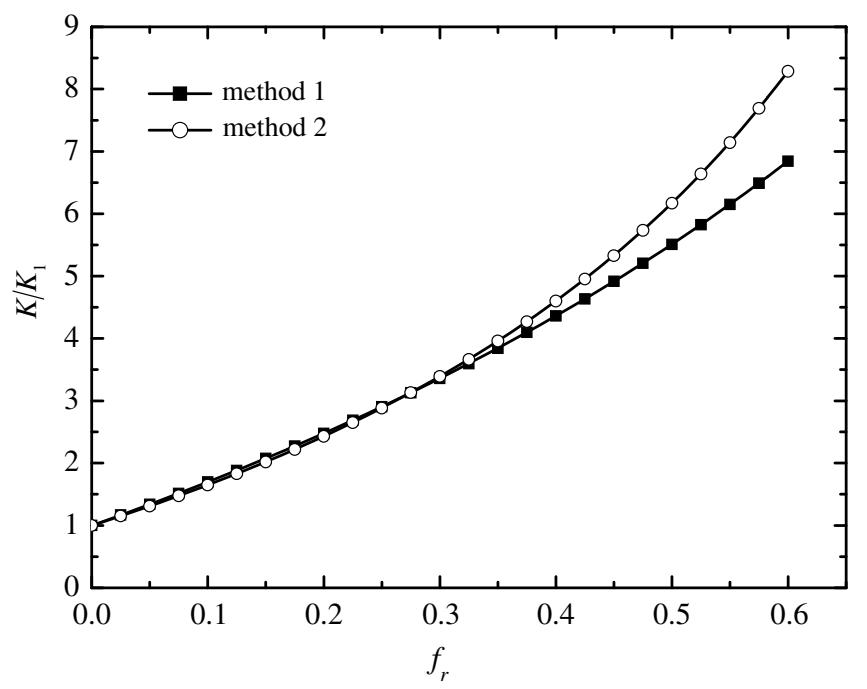

Fig. 11. Variation of effective bulk modulus with volume fraction $(x=0.2, y=0.5)$.

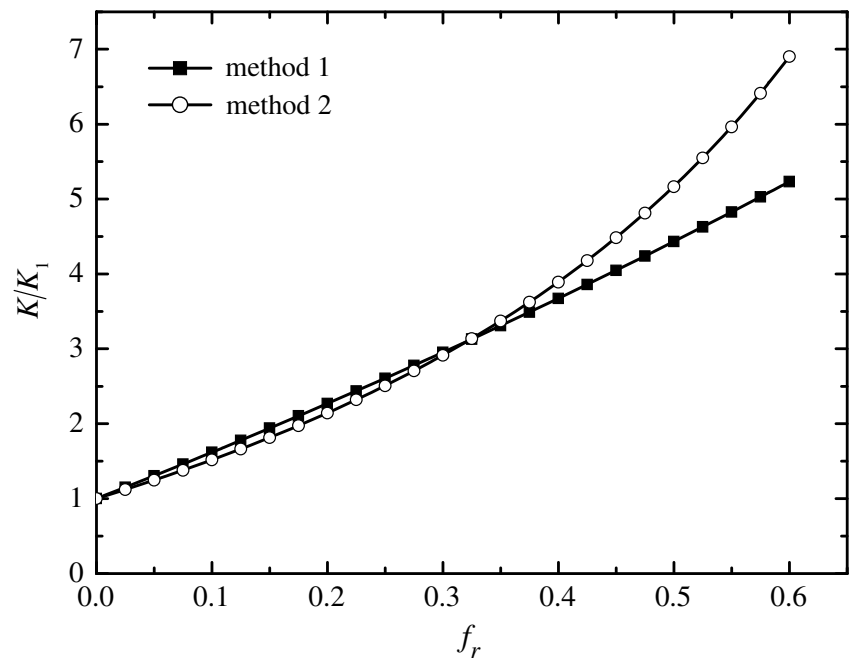

Fig. 12. Variation of effective bulk modulus with volume fraction $(x=0.2, y=0)$.

method can be used to analyze the effect of debonding on the effective elastic moduli of the composites.

\section{Conclusions and discussion}

Waviness of carbon nanotubes and interfacial bonding conditions play a critical role in determining their reinforcing efficiency in CNT-reinforced nanocomposites. The studies of these effects on the mechanical properties are of highly theoretical and technological significance for both mico-size and nano-size fibre-reinforced composites. In this paper, the influences of the waviness and debonding of carbon nanotubes on the effective elastic moduli of the composites are investigated. The proposed methods are very simple and easy to use. It is shown that both the waviness and debonding can significantly reduce the stiffening effect of the nanotubes, though they have exceptionally high modulus. In particular, the effective elastic constants of the composites are very sensitive to the waviness when the latter is small. Nevertheless, the load-transferring mechanisms at the interface between the nanotubes and the matrices may be very complicated, and both waviness and debonding may exist simultaneously. Thus, more detailed studies are needed in understanding and predicting the mechanical properties of carbon nanotube-reinforced nanocomposites.

\section{Acknowledgements}

This work is supported by the Engineering Research Institute of Peking University and the NNSFC through Grant No. 10525209.

\section{References}

[1] Thostenson ET, Ren Z, Chou TW. Advances in the science and technology of carbon nanotubes and their composites: a review. Compos Sci Tech 2001;61:1899-912. 
[2] Chronakis IS. Novel nanocomposites and nanoceramics based on polymer nanofibers using electrospinning process - a review. J Mater Proc Tech 2005;167:283-93.

[3] Cha SI, Kim KT, Lee KH, Mo CB, Hong SH. Strengthening and toughening of carbon nanotube reinforced alumina nanocomposite fabricated by molecular level mixing process. Scr Mater 2005;53:793-7.

[4] Qian D, Dickey EC, Andrews R, Rantell T. Load transfer and deformation mechanisms in carbon nanotube-polystyrene composites. Appl Phys Lett 2000;76:2868-70.

[5] Lau K-T, Lu M, Liao K. Improved mechanical properties of coiled carbon nanotubes reinforced epoxy nanocomposites. Composites A 2006;37:1837-40.

[6] Andrews R, Weisenberger MC. Carbon nanotube polymer composites. Curr Opin Solid State Mater Sci 2004;8:31-7.

[7] Shaffer MSP, Windle AH. Fabrication and characterization of carbon nanotube/poly (vinyl alcohol) composites. Adv Mater 1999;11:937-41.

[8] Ning JW, Zhang JJ, Pan YB, Guo JK. Fabrication and mechanical properties of $\mathrm{SiO}_{2}$ matrix composites reinforced by carbon nanotube. Mater Sci Eng A 2003;357:392-6.

[9] Fisher FT, Bradshaw RD, Brinson LC. Fiber waviness in nanotubereinforced polymer composites - I: Modulus predictions using effective nanotube properties. Compos Sci Tech 2003;63:1689-703.

[10] Shi DL, Feng XQ, Huang YGY, Hwang KC, Gao HJ. The Effect of nanotube waviness and agglomeration on the elastic property of carbon nanotube-reinforced composites. J Eng Mater Tech 2004; 126:250-7.

[11] Anumandla V, Gibson RF. A comprehensive closed form micromechanics model for estimating the elastic modulus of nanotubereinforced composites. Composites A 2006;37:2178-85.

[12] Chan WS, Wang JS. Influence of fiber waviness on the structural response of composite laminates. J Thermoplast Compos Mater 1994;7:243-60.

[13] Hsiao HM, Daniel IM. Elastic properties of composites with fiber waviness. Composites A 1996;27:931-41.

[14] Christensen RM, Waals FM. Effective stiffness of randomly oriented fiber composites. J Compos Mater 1972;6:518-32.

[15] Salvetat JP, Bonard JM, Thomson NH, Kulik AJ, Forr'o L, Benoit W, et al. Mechanical properties of carbon nanotubes. Appl Phys A 1999;69:255-60.

[16] Li C, Chou TW. Multiscale modeling of carbon nanotube reinforced polymer composites. J Nanosci Nanotech 2003;3:423-30.

[17] Wagner HD. Nanotube-polymer adhesion: a mechanics approach. Chem Phys Lett 2002;361:57-61.

[18] Baney JM, Zhao YH, Weng GJ. Progressive debonding of aligned oblate inclusions and loss of stiffness in a brittle matrix composite. Eng Fract Mech 1996;53:897-910.
[19] Zhao YH, Weng GJ. Transversely isotropic moduli of two partially debonded composites. Int J Solids Struct 1997;34:493-507.

[20] Odegarda GM, Gatesb TS, Wisea KE, Park C, Siochi EJ. Constitutive modeling of nanotube-reinforced polymer composites. Compos Sci Tech 2003;63:1671-87.

[21] Thostenson ET, Chou TW. On the elastic properties of carbon nanotube-based composites: modeling and characterization. J Phys D Appl Phys 2003;36:573-82.

[22] Gao XL, Li K. A shear-lag model for carbon nanotube-reinforced polymer composites. Int J Solids Structures 2005;42:1649-67.

[23] Chou TW, Takahashi K. Non-linear elastic behavior of flexible fibre composites. Composites 1987;18:25-34.

[24] Kuo CM, Takahashi K, Chou TW. Effect of fiber waviness on the nonlinear elastic behavior of flexible composites. J Compos Mater 1988;22:1004-25.

[25] Mori T, Tanaka K. Average stress in matrix and average energy of materials with misfitting inclusions. Acta Metall 1973;21:571-4.

[26] Chen T, Dvorak GJ, Benveniste Y. Mori-Tanaka estimate of the overall elastic moduli of certain composite materials. J Appl Mech 1992;59:539-46.

[27] Wang J, Pyrz R. Prediction of the overall moduli of layered silicatereinforced nanocomposites - Part I: basic theory and formulas. Compos Sci Tech 2004;64:925-34.

[28] Zhang H, Ericson ML, Varna J, Berglund LA. Transverse single-fibre test for interfacial debonding in composites: 1. Exp Observations Compos A 1997;28:309-15.

[29] Yu Q. Fracture toughness of composite materials reinforced by debondable particulates. Scr Mater 2003;49:491-6.

[30] Zuiderduin WCJ, Westzaan C, Huetink J, Gaymans RJ. Toughening of polypropylene with calcium carbonate particles. Polymer 2003;44:261-75.

[31] Tvergaard V. Debonding of short fibres among particulates in a metal matrix composite. Int J Solids Struct 2003;40:6957-67.

[32] Hill R. Theory of mechanical properties of fibre-strengthened materials I: elastic behaviour. J Mech Phys Solids 1964;12: 199-212.

[33] Walpole LJ. Elastic behaviour of composite materials: theoretical foundations. In: Yih Chia-Shun, editor. Advances in applied mechanics, vol. 21. New York: Academic Press; 1981.

[34] Shao LH. Study of mechanical properties of carbon nanotubereinforced composites with debonding and waviness. MSc Thesis, College of Engineering, Peking University, 2007.

[35] Wu JF, Bai S, Liu SH, Xu HJ, Cheng HM. Fabrication and characterization of large isotropic pyrolytic carbons. New Carbon Mater 2006;21:119-24 [in Chinese].

[36] Su JM, Yang J, Xiao ZC, Zhou SJ, Peng ZG, Xin JG, et al. Structure and properties of carbon/carbon composite materials for aircraft brake discs. New Carbon Mater 2006;21:81-9 [in Chinese]. 